\title{
A physiotherapy-led transition to home intervention for older adults following emergency department discharge: protocol for a pilot feasibility randomised controlled trial
}

Mairéad Conneely ${ }^{1 *} \mathbb{0}$, Aoife Leahy ${ }^{1,2}$, Margaret O'Connor ${ }^{2}$, Louise Barry ${ }^{3}$, Gillian Corey ${ }^{3}$, Anne Griffin ${ }^{1}$, Íde O'Shaughnessy ${ }^{1,2}$, Ida O'Carroll ${ }^{2}$, Siobhán Leahy ${ }^{4}$, Dominic Trépel ${ }^{5}$, Damian Ryan ${ }^{6}$, Katie Robinson ${ }^{1}$ and Rose Galvin ${ }^{1}$

\begin{abstract}
Background: Older adults frequently attend the emergency department (ED) and experience high rates of adverse outcomes following ED presentation including functional decline, ED re-presentation and unplanned hospital admission. The development of effective interventions to prevent such outcomes is a key priority for research and service provision. This paper reports a protocol designed to evaluate the feasibility of conducting a three arm randomised controlled trial (RCT) within the ED setting and in the patient's home. The interventions are comprehensive geriatric assessment (CGA), ED PLUS and usual care.

Methods: The ED PLUS pilot trial is designed as a feasibility RCT conducted in the ED and Acute Medical Assessment Unit of a university teaching hospital in the mid-west region of Ireland. We aim to recruit 30 patients, aged 65 years and over presenting to the ED with undifferentiated medical complaints and discharged within $72 \mathrm{~h}$ of index visit. Patients will be randomised by a computer in a ratio of 1:1:1 to deliver usual care, CGA or ED PLUS during a 6-month study period. A randomised algorithm is used to perform randomization. CGA will include a medical assessment, medication review, nursing assessment, falls assessment, assessment of mobility and stairs, transfers, personal care, activities of daily living (ADLs), social supports and baseline cognition. ED PLUS, a physiotherapist led, multidisciplinary intervention, aims to bridge the transition of care between the index visit to the ED and the community by initiating a CGA intervention in the ED and implementing a 6-week follow-up self-management programme in the patient's own home following discharge from the ED. The outcomes will be parameters of the feasibility of the intervention and trial methods and will be assessed quantitatively and qualitatively.
\end{abstract}

\footnotetext{
*Correspondence: mairead.c.conneely@ul.ie

${ }^{1}$ School of Allied Health, Faculty of Education and Health Sciences,

Ageing Research Centre, Health Research Institute, University of Limerick, Limerick, Ireland

Full list of author information is available at the end of the article
}

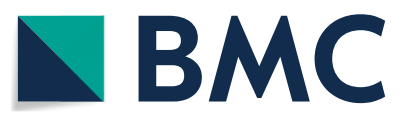

(c) The Author(s) 2021. Open Access This article is licensed under a Creative Commons Attribution 4.0 International License, which permits use, sharing, adaptation, distribution and reproduction in any medium or format, as long as you give appropriate credit to the original author(s) and the source, provide a link to the Creative Commons licence, and indicate if changes were made. The images or other third party material in this article are included in the article's Creative Commons licence, unless indicated otherwise in a credit line to the material. If material is not included in the article's Creative Commons licence and your intended use is not permitted by statutory regulation or exceeds the permitted use, you will need to obtain permission directly from the copyright holder. To view a copy of this licence, visit http://creativecommons.org/licenses/by/4.0/. The Creative Commons Public Domain Dedication waiver (http://creativeco mmons.org/publicdomain/zero/1.0/) applies to the data made available in this article, unless otherwise stated in a credit line to the data. 
Discussion: Rising ED visits and an ageing population with chronic health issues render ED interventions to reduce adverse outcomes in older adults a research priority. This feasibility RCT will generate data and experience to inform the conduct and delivery of a definite RCT.

Trial registration: The trial was registered in Clinical Trials Protocols and Results System as of $21^{\text {st }}$ July 2021, with registration number NCT049836020.

Keywords: Older adults, Emergency department, Adverse outcomes, Integrated care, Feasibility randomised controlled trial

\section{Background}

Population ageing poses challenges to the healthcare system with older adults requiring acute health care services at an increasing rate [1], particularly for emergency department (ED) services [2-5]. EDs are complex environments in which to provide care to older adults $[6,7]$. Older adults are more likely to experience longer ED lengths of stay, have more complex presentations and higher rates of adverse outcomes following discharge from the ED than younger people [8-10]. Adverse outcomes include functional decline, poorer quality of life, unscheduled return visits to the ED, hospitalisation and mortality [11-13]. Given the risk of adverse outcomes following an index visit, the development of effective interventions is a key priority for research and service provision.

A presentation to an ED can be viewed as an opportunity to assess those at risk of adverse outcomes and initiate a care plan in those deemed as 'high risk' [2, 14]. Consequently, a number of interventions have been examined to reduce the incidence of adverse outcomes among 'high risk' older adults following presentation to the ED [15-18]. The evidence from systematic reviews regarding the effectiveness of these interventions is mixed given the heterogeneity in study designs, description of the interventions and outcomes assessed. In line with Medical Research Council (MRC) Guidelines $[19,20]$, we developed an evidence base by completing an umbrella review of the effectiveness of ED initiated interventions to improve outcomes in older adults. This comprehensive umbrella review included nine systematic reviews representing 29 randomised controlled trials (RCTs) investigating various interventions including implementation of care pathways based on risk profiling, nurse-led interventions, case management within the ED and post-discharge, comprehensive geriatric assessment (CGA) and discharge planning [17]. These interventions were either ED-based interventions or ED-initiated interventions with community follow up, termed EDcommunity transitional services interventions $[16,17]$. This umbrella review identified low-quality evidence to support ED interventions in reducing functional decline, improving patient experience and improving quality of life. The quality of evidence of the effectiveness of ED interventions to reduce mortality and ED revisits varied from very low to moderate. There was no effect of any of the interventions on hospital admissions after the ED index visit. No systematic review reported the outcome of length of ED stay. The authors of all systematic reviews included in this umbrella review recommend that more high quality RCTs need to be conducted in this area.

One of the most commonly investigated interventions in older adults is CGA, which is considered the gold standard approach to improving a range of outcomes for frail older adults in acute hospitals [21]. There is evidence that a treatment plan based on CGA can increase the chance of living independently at home, and have a positive effect on physical function outcomes compared to usual care in various other care settings, including hospitalised older adults [21-23], but the evidence base for its implementation in the ED is insufficient $[24,25]$. Thus, clinicians have limited evidence on which to base their practice. There is a need for further pragmatic trials to assess the effectiveness of CGA in an ED setting.

Our umbrella review did not identify any systematic review that explored an ED intervention targeting older adults led by health and social care professionals (HSCPs) such as physiotherapists, medical social workers and occupational therapists. Research has demonstrated that HSCPs can play a role in the ED in improving patient experience, reducing length of ED stay and preventing hospital admissions in other age groups [26-28].

HSCPs are well placed to deliver interventions to reduce functional decline among older adults. Furthermore, physiotherapists are key HSCPs who lead interventions to improve functional decline and address other adverse outcomes in hospital and community settings [13, 29-31]. Integrated care programmes which include screening to identify needs, linking to community services and monitoring with a primary care provider, have been advocated to improve the continuum of care from the ED into the community [32-35].

A key research priority defined by Kings Fund and Veteran Affairs Reports is to develop user informed transitional interventions to bridge the ED and community [16]. We developed a novel intervention in response to 
the findings from our umbrella review to address service needs and key geriatric research priorities outlined in the literature [36-39]. The proposed intervention, ED PLUS, is a user-informed, self-management programme developed based on best available evidence to improve functional decline in older adults. ED PLUS, a physiotherapist-led, multidisciplinary intervention, bridges the transition of care between the index visit to the ED and the community by initiating a CGA intervention in the ED and implementing a 6-week follow-up self-management programme in the patient's own home following discharge from the ED. This feasibility RCT will generate data and experience to inform the conduct and delivery of a definite RCT.

\section{Aims and objectives}

The overall aim of this work is to examine the feasibility of implementing a 6-week transitional intervention delivered both face to face and via telephone support for older adults discharged from the ED.

Primary aims are:

1. To establish whether the intervention is acceptable to older adults discharged from the ED

2. To assess the feasibility of undertaking a definitive RCT of the ED PLUS intervention in this population, evaluating the process of recruitment, screening, randomisation, and collection of data at baseline and outcome data

3. To explore any trial design aspects that may require refinement prior to proceeding to a full RCT

Secondary aims are:

1. To examine the effect of ED PLUS on functional decline at 6 weeks and 6 months
2. To explore the effect of the ED PLUS on quality of life at 6 weeks and 6 months

3. To examine the effects of ED PLUS on process outcomes (ED revisits, unplanned admission, length of stay) at 6 weeks and 6 months

\section{Methods}

Trial design

This is a parallel group pilot RCT with a 1:1:1 allocation ratio. In order to ensure standardised conduct and reporting, the Standard Protocol Items for Intervention Trials (SPIRIT) guidelines will be used [40]. Table 1 describes the feasibility/piloting phase of the MRC Framework alongside the activities involved in this process evaluation [41]. The trial is registered at Clinical trials.gov (NCT04983602). Ethics approval was obtained from the HSE Mid-Western Area Research Ethics Committee $(088 / 2020)$.

\section{Trial setting}

The pilot RCT will be conducted in the ED and Acute Medical Assessment Unit (AMAU) of a university teaching hospital in the mid-west region of Ireland. University Hospital Limerick (UHL) serves both rural and urban areas of Limerick, Clare and North Tipperary. This is a university teaching hospital, which caters for the general medical, surgical and emergency treatment of patients in its catchment area of 470,000 people. UHL has a $24 \mathrm{~h}$, seven days a week ED that functions 365 days a year and is a tertiary referral centre for the mid-west region.

\section{Participants \\ Sample size}

As this is a pilot feasibility RCT, the primary aim is not to evaluate the clinical effectiveness; therefore, we will not

Table 1 Mapping activities to Medical Research Council framework section 2 assessing feasibility and pilot methods

\begin{tabular}{|c|c|c|}
\hline 2 & Assessing feasibility and piloting methods & \\
\hline 2.1 & $\begin{array}{l}\text { Testing procedures for acceptability, compliance, and } \\
\text { intervention delivery }\end{array}$ & $\begin{array}{l}\text { Tested components for feasibility and acceptability. } \\
\text { Acceptability of the ED PLUS intervention discussed with PPI panel. } \\
\text { Assess feasibility of delivering intervention via face-to-face interven- } \\
\text { tion and via telephone in terms of recruitment, retention and usability } \\
\text { through a pilot of } 6 \text { weeks with } 10 \text { participants. } \\
\text { Assess acceptability through qualitative interviews. }\end{array}$ \\
\hline 2.2 & Estimating recruitment and retention & $\begin{array}{l}\text { Recruitment from an ED and AMAU of a large, single tertiary care facility. } \\
\text { Consult with PPI group of older adults to determine best practice for } \\
\text { ongoing retention of trial participants. } \\
\text { Consult with trial methodology groups (e.g. Health Research Board Trial } \\
\text { Methodology Research Network) and working groups to determine the } \\
\text { best methodology for ongoing retention of trial participants. }\end{array}$ \\
\hline 2.3 & Determining sample size & The results will be used to inform the sample size of a future definite RCT. \\
\hline
\end{tabular}


undertake a formal power analysis for sample size for a primary outcome [42, 43]. We aim to recruit 30 participants over a two-month period as recommended for pilot studies $[44,45]$. From other studies in similar settings, we anticipate a drop-out rate of $10 \%$ in total for the three groups $[46,47]$. Data obtained from this pilot RCT will inform the power analysis for a definitive trial.

\section{Eligibility criteria}

The inclusion criteria for participants are adults aged over 65 years of age with undifferentiated medical complaints presenting to an ED. Participants must:

- Be medically stable as deemed by the treating physician (vital signs are within normal limits, patients do not require a surgical assessment) [48]

- Have a score of $\geq 2$ on the Identification of Seniors at Risk (ISAR) screening tool $[49,50]$. The ISAR is a validated screening tool for use in the ED to detect older adults at risk of adverse outcomes including functional decline, revisits to the ED, unplanned hospitalisation and mortality within 6 months of the ED presentation. A cut-off of $\geq 2$ is recommended [49].

- Be community dwelling

- Have a short-term ED admission with a length of stay of $\leq 72 \mathrm{~h}$ from ED presentation.

Exclusion criteria include:

- Individuals under the age of 65 years

- Have a score of $<12$ on the ISAR

- Older adults who present with acute myocardial infarction, stroke or non-medical problems or require a surgical assessment or psychiatric issues including schizophrenia

- Older adults who are medically unstable

- If neither the patient nor carer can communicate in English sufficiently to complete consent or baseline assessment

- Older adults who are admitted to hospital from the ED Older adults who have a confirmed positive COVID-19 test on presentation to the ED

\section{Recruitment}

Patients will be screened for inclusion between 8 am and 4 pm Monday to Friday. Older adults who score $\geq 2$ on the ISAR will be asked if they would like to participate in the trial by a dedicated research nurse during their ED or AMAU visit. They will be given an opportunity to read the information leaflet and ask any questions they may have before giving written informed consent if agreeable. Potential participants will be approached regarding trial recruitment post ED triage thereby ensuring rapid assessment shortly after hospital arrival by the dedicated SOLAR team in the ED. The SOLAR team (consisting of a consultant in geriatric medicine/geriatric specialist registrar, specialist geriatric nurse, senior pharmacist, senior physiotherapist, senior occupational therapist and senior medical social worker) in the ED will assess all participants in the intervention group and perform CGA.

Figure 1 outlines the trial design.

\section{Randomisation and allocation concealment}

Eligible participants will be block randomised in groups of 30 participants using a computer generated 1:1:1 allocation through a Sealed Envelope website (www.seale denvelope.com). Allocation will be concealed from participants and researchers until after consent has been obtained and baseline evaluations performed. The research nurse (GC) will assign participants to interventions once allocation has been revealed.

\section{Blinding}

Due to the nature of the intervention, trial participants and treating clinicians are unable to be blinded to group allocation. The outcome assessor will be blinded to group allocation. The outcome assessor has no access to the medical files of the participants and will be given a list of questions related to the outcome measures to ask each participant at 6 weeks and 6 months.

\section{Informed consent}

Potential participants will initially be informed of the study by the triage nurse or the treating physician. If participants indicate a willingness to hear more about the trial, the dedicated research nurse (GC) on the project will be informed. The research nurse will subsequently meet with the potential participant, describe the study in detail and provide potential participants with an information leaflet. Participants will be given time to consider the trial and to ask questions. Written informed consent will be obtained by the research nurse if/when the participant indicates their willingness to formally participate in the study. Participants will have the duration of their index admission to consider participation in the trial. Participants can withdraw at any stage without any negative implications for their treatment.

\section{Interventions}

There are three arms to this pilot feasibility trial: usual care, intervention arm 1 (CGA) and intervention arm 2 (ED PLUS). 


\section{ED Index Visit}

Eligibility Screening

Written Consent and Baseline Assessment (T1)

Randomisation

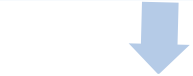

\section{Group Allocation}

\section{Interventions}

\section{Post-Intervention Assessment (T2)}

Follow-Up Assessment (T3)

Qualitative Evaluation

Fig. 1 Study design 


\section{Usual Care}

The comparison group will receive routine care as would be usual in the ED. At present, there is no dedicated team to perform CGA in the ED and AMAU at UHL, but an interdisciplinary HSCP team is available at the discretion of the referring ED doctor or medical team. This process will be continued during the study and will be documented.

\section{Intervention arm 1 comprehensive geriatric assessment}

The intervention arm 1 will comprise initially of a detailed interdisciplinary assessment and intervention by one or more members of the dedicated SOLAR team in UHL.

Comprehensive geriatric assessment (CGA) will include but not be limited to a medical assessment, medication review, nursing assessment, falls assessment, assessment of mobility and stairs, transfers, personal care, activities of daily living (ADLs), social supports and baseline cognition. Members of the SOLAR team will be guided by their clinical expertise and codes of professional practice. Similarly, interventions prescribed by the SOLAR team will be based on subjective and objective assessment of patients and will include medication alterations, lifestyle advice, prescription of mobility aids, provision of home exercise programmes and onward community referral as appropriate. All assessments and interventions will be included in the medical chart of individual participants. All healthcare professionals will adhere to guidelines for COVID-19 throughout the duration of the intervention. Table 2 outlines the core components of CGA.

\section{Intervention arm 2 ED PLUS}

The ED PLUS programme consists of CGA in the ED plus a 6-week multifactorial, multidisciplinary, patient centred self-management support and exercise programme. The team consists of a geriatrician, dietitian, occupational therapist and led by a physiotherapist. It is structured to maximise the patient's self-efficacy and support independence in functional activities and provide a continuum of care from the ED to the patient's home. ED PLUS aims to address issues with mobility, strength, balance, malnutrition, medication adherence, fatigue and enable self-management. The development of ED PLUS has been informed by the best available theoretical evidence on the conduct of feasibility trials of complex interventions [51]. A synthesis of the literature was undertaken and input was also provided from geriatricians, geriatric nurses, dietitians, occupational therapists, physiotherapists and a PPI panel of older adults. Implementing care to older adults is complex due to the presence of multimorbidity
Table 2 Components of Comprehensive Geriatric Assessment

\begin{tabular}{ll}
\hline Medical & Co-morbid conditions and disease severity \\
& Medication review \\
& Nutritional status \\
& Problem list \\
Mental health & Cognition \\
& Mood and anxiety \\
& Fears \\
Functional capacity & Basic activities of daily living \\
& Gait and balance \\
& Activity/exercise status \\
& Instrumental activities of daily living \\
Social circumstances & Informal support from family or friends \\
& Social network such as visitors or daytime activities \\
& Eligibility for being offered care resources \\
& Home comfort, facilities and safety \\
Environment & Transport facilities \\
& Accessibility to local resources
\end{tabular}

[37]. This programme is designed with the latest evidence base on the management of people with multiple chronic conditions [52], in particular a 6-week occupational therapy-led, multidisciplinary, self-management programme for community dwelling people with multimorbidity [53]. Furthermore, the existing evidence base supports the appointment of one clearly defined health care professional who co-ordinates care, a focus on patient preferences, shared decision making and a focus on functional outcomes [52]. An intervention co-ordinated by a defined HSCP has shown to be to be successful in improving clinical outcomes among frequent ED users [54].

The 6-week programme will involve a telephone call from the discharging member of the SOLAR team in the ED to the lead physiotherapist (MC) who will subsequently contact the patient to arrange a physiotherapist home visit. The home visit will take place $24-48 \mathrm{~h}$ post ED discharge and involve an initial assessment and the setting of patient goals over the 6-week period. The programme will involve three home visits by a physiotherapist over a period of 6 weeks with telephone support in between the visits by the physiotherapist. The other members of the MDT will telephone the patient as outlined in Fig. 2. The physiotherapist will implement a personalised treatment plan liaising with the geriatrician with key regard to medication adherence, exercise prescription, physical activity and strength. ED PLUS incorporates the most recent evidence on physical activity and falls prevention, duration of physical activity programmes, management of multimorbidity and physical exercise interventions for improving measures of physical function in older adults [31, 52, 55-57]. The evidence for the benefits of exercise in improving age-related decline and preventing falls are significant [56]. Evidence exists 


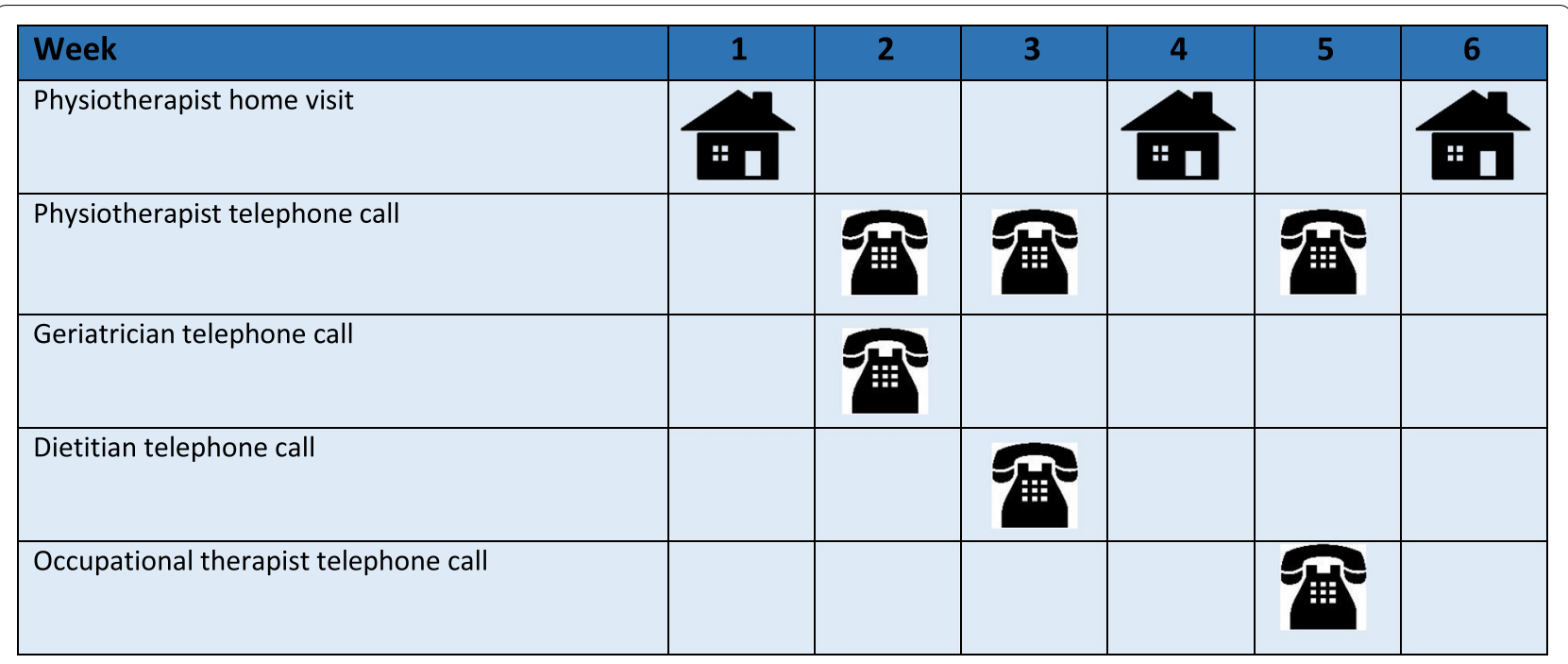

Fig. 2 Frequency of ED PLUS sessions

that physiotherapists help prevent falls following acute rehabilitation [58] with The New Physical Activity Guidelines for Health in the UK have strength and balance activities recommended for older adults who are frail [59].

As outlined in Fig. 2, the geriatrician will conduct a telephone call with the participants to discuss medication, medication adherence and address concerns regarding same. The dietitian will offer a telephone one-to-one dietetic sessions to optimise dietary intake, ensuring adequate energy, protein and micronutrient status given the relationship of malnutrition and functional status [33, $60,61]$. There is also evidence of a link between malnutrition and poorer quality of life, increased risk of hospital admissions and a greater likelihood of admission [62]. The occupational therapist will discuss with the patient regarding self-management including management of morbidity, fatigue and energy conservation to ensure ongoing engagement in physical activity programmes and leisure pursuits. The physiotherapist will act as the key case worker and support the patient during the 6-week programme to address any issues related to their health with liaison with the patient's doctor as required. Healthcare professionals will adhere to guidelines for COVID 19 throughout the duration of the intervention. Table 3 outlines the ED PLUS intervention and Fig. 2 illustrates the frequency of the sessions.

\section{Patient and public involvement}

The programme was developed in conjunction with a local public and patient involvement (PPI) panel of older adults in the mid-west/UHL catchment area; this panel was set up to support researchers in geriatric emergency medicine research [63]. To ensure the relevance of research outcomes, it is essential that the views and experiences of older people are taken into account when designing research [16]. The lead author (MC) conducted telephone conversations with representatives from the panel $(N=5)$ to initiate interest in such a trial, assist in the formation of patient centred outcomes, inform the best mode of delivery and the preparation of a patient information booklet. The PPI panel of older adults advised of conducting the physiotherapy assessment in the patients' home as soon as possible post discharge. The panel also concurred with evidence on a key HSCP to co-ordinate care and to limit the number of HSCPs visiting the home. In order to foster independence, the panel recommended a combination of face-to-face sessions as well as telephone support for patients.

\section{Criteria for discontinuing or modifying allocated interventions}

CGA and ED PLUS are dynamic processes and may change depending on the patient's presentation. Participation in the trial is voluntary. Participants will be free to withdraw consent and leave the study at any time without giving a reason and without affecting their care. Should a patient withdraw consent to participate in the study, we will seek clarification on whether withdrawal is from a particular element of the study, for example, participation in the CGA, baseline data assessment or access to healthcare records. Data will be irrevocably deleted from the database, in line with participants' choice of withdrawal. In cases where participants do not respond to follow-up assessments, outcome data that do not involve 
Table 3 Components of ED PLUS

\begin{tabular}{|c|c|c|}
\hline $\begin{array}{l}\text { Administration } \\
\text { timeline }\end{array}$ & Personnel & Description \\
\hline Week 1 & Physiotherapist visiting the patients home & $\begin{array}{l}\text { Physiotherapy assessment and action plan including individual- } \\
\text { ized goal setting based on the CGA conducted in the ED and } \\
\text { the physiotherapy assessment. The physiotherapist performs an } \\
\text { assessment of gait, balance, upper and lower limb strength assess- } \\
\text { ment, assesses the patients ability to function independently. An } \\
\text { exercise programme is tailored to the patient (examples attached) } \\
\text { https://www.nhsinform.scot/healthy-living/preventing-falls/keepi } \\
\text { ng-well/strength-and-balance-exercises } \\
\text { https://theros.org.uk/information-and-support/osteoporosis/ } \\
\text { living-with-osteoporosis/exercise-and-physical-activity-for-osteo } \\
\text { porosis/ }\end{array}$ \\
\hline Week 2 & $\begin{array}{l}\text { Medication assessment and medication action plan } \\
\text { Via telephone } \\
\text { Physiotherapist via telephone call }\end{array}$ & $\begin{array}{l}\text { The geriatrician will assess the medication the patient is taking as } \\
\text { well as make recommendations for tapering of medication and } \\
\text { cessation. } \\
\text { Physiotherapist will advise on progressions of exercise programme } \\
\text { as appropriate. }\end{array}$ \\
\hline Week 3 & $\begin{array}{l}\text { Nutritional assessment with a dietitian via telephone. } \\
\text { Physiotherapist via telephone call }\end{array}$ & $\begin{array}{l}\text { Nutritional status will be determined using validated screening } \\
\text { tools and a nutritional care plan will be implemented. } \\
\text { Physiotherapist will advise on progressions of exercise programme } \\
\text { as appropriate. }\end{array}$ \\
\hline Week 4 & Physiotherapist visiting the patients home & $\begin{array}{l}\text { The physiotherapist reassess the patient and progresses as appro- } \\
\text { priate, discuss any concerns with the patient and action plan as } \\
\text { appropriate }\end{array}$ \\
\hline Week 5 & $\begin{array}{l}\text { Occupational Therapist via telephone } \\
\text { Physiotherapist via telephone call }\end{array}$ & $\begin{array}{l}\text { The occupational therapist (OT) will discuss with the patient } \\
\text { regarding self-management based on the individual goals set by } \\
\text { the patient and physiotherapist. These strategies may include: } \\
\text { - Fatigue and energy management } \\
\text { - Managing stress and anxiety and maintaining mental health and } \\
\text { well-being } \\
\text { Physiotherapist will advise on progressions of exercise programme } \\
\text { as appropriate. }\end{array}$ \\
\hline Week 6 & $\begin{array}{l}\text { Physiotherapist will visit patients home to conclude the sessions } \\
\text { with a focus on review of patient goals and action plan to follow. }\end{array}$ & $\begin{array}{l}\text { Input from all HSCPs is collated for each patient to conclude their } \\
\text { ED PLUS management. }\end{array}$ \\
\hline
\end{tabular}

participant contact (e.g. data from hospital database) will continue to be collected in these cases.

\section{Strategies to improve adherence to interventions}

A number of strategies have been implemented to support adherence to the intervention. A detailed protocol has been developed and shared with the SOLAR team. Daily briefings/team meetings are planned to troubleshoot queries or concerns by the team. Training on the procedures around participant consent has been conducted. A comprehensive and detailed delegation log has been developed. The ED PLUS programme was developed with the PPI group of older adults regarding the delivery of the intervention and the content thus this is a user-informed intervention as per best practice [16].

\section{Relevant concomitant care permitted or prohibited during the trial}

Participants will be under the medical care of their treating physician for the duration of their ED stay. All relevant consultant stakeholders will continue to provide clinical governance for the patient as per standard clinical practice.

\section{Provisions for post-trial care}

A detailed letter describing the ED PLUS programme will be sent to the general practitioner (GP) and other HSCPs as required for all intervention group participants discharged directly from the ED. All other patients will have the usual hospital discharge letter provided on discharge.

\section{Data collection and outcomes}

The following data will be collected during the trial:

1. Feasibility outcomes: data from screening, recruitment and follow-up logs will be used to generate realistic estimates of eligibility, recruitment, consent and follow-up rates.

Feasibility will be described in term of recruitment rates, adherence, retention and acceptability of ED PLUS. Recruitment rates will be described at the percentage 
of eligible study population who consent to participate in ED PLUS. Adherence will be recorded as the number of home visits made by the physiotherapist in the ED PLUS team, plus the number of interactions with the geriatrician, dietician, physiotherapist and occupational therapist.

Retention will be defined as the percentage of enrolled participants completing the post-intervention assessment at 6 weeks. Acceptability of the intervention will be determined through the qualitative evaluation interviews. For the ED PLUS, face to face visits will be recorded and adherence will be calculated as a percentage of the total number of visits. These data alongside the qualitative interviews will be used to measure adherence and to assess whether any modifications are required to improve engagement.

2. Clinical outcome measures

1. Functional status using the Barthel Index [64]. The Barthel Index is the most commonly used tool for assessment of functional outcome in older adults in clinical settings $[65,66]$

2. Quality of life using the EuroQoL-5D EQ-5D-5L [67], a standardised measure of health status

3. ED revisit assessed via medical records and patient recall and nursing home admission within 6 weeks and 6 months of initial index visit

4. Unplanned hospitalisation nursing home admission assessed via patient recall and medical records within 6 weeks and 6 months of initial index visit

5. Mortality

6. Healthcare utilisation will also be captured at 6 weeks by telephone contact

Follow-up at 6 weeks and 6 months will be captured by telephone contact by a research nurse who is blinded to group allocation (Fig. 3).

\section{Economic evaluation}

Given the stage of development of ED PLUS, economic evaluation will primarily seek to examine and help establish resources required for this complex intervention. This early-stage economic evaluation will work alongside the pilot feasibility RCT and will measure resource intensity, will help establish important intangibles (e.g. non face-to-face time related to intervention delivery) and will determine correct ways to assign salient costs to resources consumed in the novel care pathway. The economic evaluation will also seek insights from qualitative research to better understand opportunity cost related

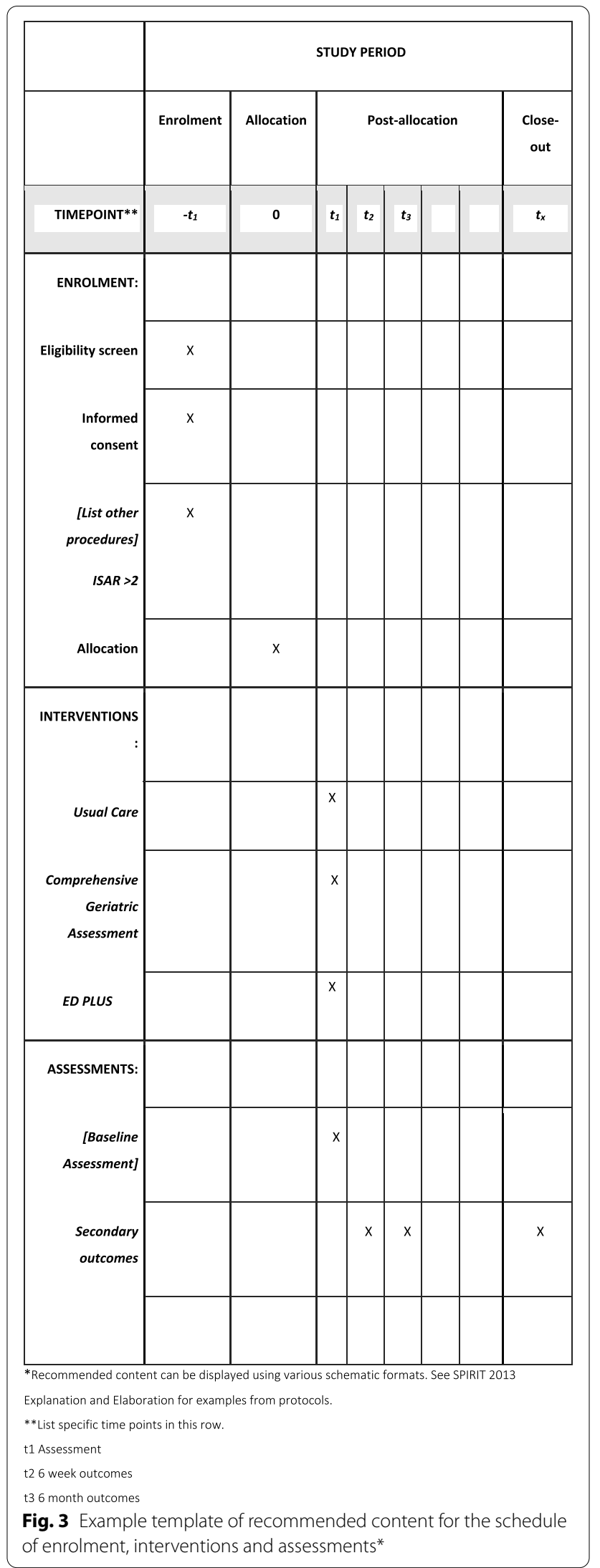


to alternative care pathway. Finally, to help develop an evidence-informed care novel care pathway that is likely to be cost effective, the economic evaluation will bring together insights to identify principal components that will likely contribute the greatest amount towards intervention effectiveness. Working with the economist, resources related to 'effective components' will be considered and recommended for future intervention refinements.

Beyond assisting with development of this complex intervention, the economic evaluation of the ED PLUS intervention will be developed to include cost effectiveness analysis. The evaluation will aim to be conducted in a manner consistent with the guidelines published by the Health and Information Quality Authority in Ireland [68]. The objectives of the evaluation will be to identify, quantify and compare the cost and effect of the ED PLUS intervention relative to those under the status quo provision of care (i.e. usual care) and CGA alone (i.e. recommended best practice).

The incremental costs and effects of the programme relative to usual care will be calculated through incremental cost effectiveness analysis and will aim to calculating incremental cost-effectiveness ratio (ICER) [59]. The ICER is formally denoted by the following:

$$
\mathrm{ICER}=\frac{\mathrm{COST}_{\mathrm{EDPlus}}-\mathrm{COST}_{\text {UsualCare }}}{\mathrm{EFFECT}_{\mathrm{EDPlus}}-\mathrm{EFFECT}_{\text {UsualCare }}}
$$

For a robust estimate of cost effectiveness, COST and EFFECTS require mean averages estimated from definitive intervention study (which will be beyond the scope of this stage of research). Furthermore, it should be noted that COST should reflect the total net cost related to care and therefore is the sum of cost of intervention (i.e. providing ED PLUS, Usual Care or CGA) plus the cost consequences elsewhere (i.e. increases or decreases in service-use related to the treatment decision).

Collection of salient cost consequences will be piloted to inform the feasibility study by collecting data in each group at each time point using the Client Service Receipt Inventory (CSRI) questionnaire [69]. Cost consequences require a sample size calculation for cost effectiveness analysis $[19,70]$ and, whilst this pilot may provide some insights to an initial calculation, it will be recommended that a full power calculation be an intended outcome of the feasibility RCT.

\section{Qualitative evaluation}

The qualitative evaluation will assess the acceptability of the trial methods, evaluate the acceptability of the ED PLUS, identify modifications to ED PLUS and describe participants experiences of ED PLUS [71]. One-to-one semi-structured telephone interviews with the dedicated research nurse, with trial participants in intervention arm 2, all medical and HSCPs involved in the intervention arm 2 will be undertaken on completion of the programme by a person independent of the trial. Participants will be contacted to undertake a telephone interview within one week of the completion of the ED PLUS intervention. All interviews will be digitally recorded and transcribed verbatim. All participants will be offered an opportunity to view their own transcript. A sample of the interview schedule is included in Appendix 2.

\section{Data analysis}

All data will be analysed and reported in accordance with the Consolidated Standards of Reporting Trials (CONSORT) guidelines for pilot and feasibility trials [72] and the CONSORT extension for reporting of patientreported outcomes [73]. As this is a feasibility study with a relatively small sample size, formal hypothesis testing is not appropriate; rather, the purpose of any analyses will be to generate estimates to inform the planning of the definitive future trial. The analysis will be completed in two stages. Stage one will summarise the feasibility outcomes. Stage two will summarise the clinical outcomes data at 6 weeks and 6 months. As it is inappropriate to use feasibility trial data to formally test for betweengroup treatment effects, the analyses will primarily be of a descriptive nature [42, 72]. Descriptive statistics of the clinical outcomes data will be produced for each trial arm. Interval estimates of the potential intervention effects, relative to CGA and usual care only, will be produced in the form of a $95 \%$ confidence interval, to ensure that the effect size subsequently chosen for powering the definitive trial is plausible. These will refine the design characteristics of the future definitive trial.

\section{Qualitative analysis}

Interview data will be transcribed in full and analysed using a reflexive approach to thematic analysis [74] which will acknowledge and consider the centrality of researcher subjectivity. Reflexivity will enable the researcher team to consider and analyse how subjective and intersubjective elements influence the research process. Analysis will be facilitated through the use of NVIVO 12 software. When coding is complete, both participants and HSCPs will be invited to review and discuss preliminary analysis of the interview data and contribute to the process of identifying themes. Participants will also be invited to co-write/design a lay summary and infographic of the findings. Group video/phone conferences or one-to-one telephone calls will be scheduled to enable participants to contribute to analysis. 


\section{Fidelity}

We will ensure fidelity to the research protocol through several means. First, the study coordinator will review baseline enrolment data on a weekly basis. Second, the study team will review the proportion of subjects who are eligible, approached, consent to participate and enrolled on a weekly basis. Reasons for refusal are tracked to allow the study team to modify how they communicate study details when they approach the participant and for subsequent study planning. The primary investigator (PI) will hold weekly meetings with the research staff to discuss study progress and address concerns.

\section{Determining progression to the full trial}

We shall progress to a full trial application if minimum success criteria are achieved in key feasibility aims and objectives. These criteria:

- A minimum of $80 \%$ recruitment of eligible patients

- A minimum of $80 \%$ completion rate of key outcome measures (including follow-up).

\section{Trial oversight and monitoring}

Given the nature of this trial, the Trial Steering Committee (TSC) will comprise of the project manager (MC), PI (RG), key co-applicants, ED PLUS and other key external members of staff involved in the study. Specifically, the TSC will be responsible for (i) protocol development, (ii) obtaining ethical approval (iii) clinical set-up, on-going management, promotion of the study and for the interpretation and publishing of the results. The TSC will provide overall supervision of the study, in particular by monitoring study progress, and provide public, clinical, and professional advice, with pre-agreed terms of reference.

\section{Safety}

In the light of COVID-19 pandemic, additional measures to increase safety of all treating staff and participants will be ensured. All patients are screened for COVID-19 on presentation to the ED and are all tested. All participants will be screened via telephone the day before a home visit to confirm the participant and all people living with them are free from signs and symptoms of COVID-19. All research staff will follow the COVID-19 protocols within UHL and the National Protocol for workers. All clinical and research staff will be fully equipped with Personal Protective Equipment (PPE) and are accustomed to use of PPE since the commencement of the pandemic in March 2020.

\section{Data management, audit and monitoring}

All relevant data entered by a research nurse will be stored on Excel and pseudo-anonymised. The key to this pseudo-anonymisation will be kept by the PI [37]. A quality check of $20 \%$ of data will be completed by an independent researcher. If there is more than $5 \%$ of errors identified across the data entry, all data will be independently checked by the second independent researcher. For the life of the study, the pseudoanonymised data will be stored on an encrypted and password protected electronic data capture system (CASTOR). Each member of the research team that is designated the task of entering data will have their own unique login and password for this system. Hard copy study-related materials including patient data will be stored in a secure locked environment with restricted access.

The confidentiality of the data will be always ensured by the PI and all members of the research team. Identifiable data will not be disclosed to third parties, and no participant's name will appear in any of the results, as indicated in the participant's information leaflet. Each participant in the study will be assigned a numerical code to link data collected at baseline to the data collected at follow-up at 6 weeks and 6 months. Aggregate data will be pseudo-anonymised. The research team will ensure anti-virus software is installed and up to date on all devices involved in data entry. All information trafficked from the clinical site to the research database will be pseudo-anonymised with unique study-specific subject numbers. Access to the research database is managed by the PI. No personal details or identifying data will be transferred from the hospital site to external sites, where the data will be analysed. Only coded/ pseudo-anonymised data will be transferred to external sites.

\section{Dissemination plan}

The results of this feasibility trial will inform the design of the anticipated definitive trial, rather than directly inform clinical decision-making, since clinical and cost effectiveness cannot be determined at this level. On completion, the results will be submitted for publication in open access peer-reviewed journals. Our PPI panel of older adults will assist in the dissemination in non-academic frameworks. All participants will be offered a lay summary of the results and a clinical summary will be presented to the ED clinical teams. A major output will be an application for funding for a 
future definite RCT, should the criteria for progression be achieved.

\section{Discussion}

Rising ED visits and an ageing population with chronic health issues render ED interventions to reduce adverse outcomes in older adults a research priority. An ED visit is a key event which signals a deteriorating functional status [75]. Early identification in the ED [19] of older adults at risk of adverse outcomes is a key research priority. CGA is considered the gold standard for the assessment of older adults, but there is insufficient evidence to support its implementation on the ED at present. A key research priority defined by Kings Fund and Veteran Affairs Reports is to develop user informed transitional interventions to bridge the ED and community [16]. The ED PLUS intervention has been developed with the aim of addressing this important issue. One of the strengths of ED PLUS is that is has been developed with clinical practitioners, researchers and a PPI panel of older adults. Best practice guidelines recommend the need to test the feasibility and acceptability of RCT procedures prior to undertaking a definite trial. Guidelines for the management on patients during COVID 19 will be adhered to. This pilot feasibility RCT will provide important operational data into the practicality of undertaking a definite RCT including estimates of recruitment, attrition, baseline assessment scores and completion rates of the measures.

\section{Trial status}

This is version 1 of the protocol $\left(16^{\text {th }}\right.$ September 2021). Recruitment is open. Any deviations to this protocol will be submitted to the respective ethics boards and updated on Clinical Trials Protocols and Results System (clinicaltr ials.gov), and the changes will be discussed on dissemination of the results.

\begin{abstract}
Abbreviations
AMAU: Acute medical assessment unit; CGA: Comprehensive geriatric assessment; CSRI: Client Service Receipt Inventory; ED: Emergency department; GP: General practitioner; HSE: Health Service Executive; ICER: Incremental cost-effectiveness ratio; ISAR: Identification of seniors at risk; MRC: Medical Research Council; PI: Primary Investigator; PPE: Personal protective equipment; PPI: Public and patient involvement; RCT: Randomised controlled trial; UHL: University Hospital Limerick.
\end{abstract}

\section{Supplementary Information}

The online version contains supplementary material available at https://doi. org/10.1186/s40814-021-00954-5.

Additional file 1: Appendix 1. ED PLUS demographic questionnaire

Additional file 2: Appendix 2. Interview guide ED PLUS participants
Acknowledgments

The authors wish to acknowledge the contribution of the public and patient involvement group (PPI) of older adults.

Name and contact of the trial sponsor

University of Limerick, Castletroy, Co. Limerick.

\section{Authors' contributions}

$M C$ drafted the original draft of the protocol. MC and RG were major contributors in writing the protocol. MC, AL, MOC, AG, SL, IOS, DR, KR and RG designed the study. KR completed the section on qualitative evaluation. DT completed the section on economic evaluation. MC, AL, MOC, LB, GC, AG, IOS, IOD, SL, $D T, D R, K R$ and RG critically appraised and edited the manuscript. SL, DT, KR and RG acted as supervisors. MOC and RG are the guarantors of the study. All authors read and approved the final manuscript.

\section{Funding}

This work was supported by the Health Research Board (HRB) of Ireland (Health Research Board, Grattan House 67-72 Lower Mount Street, Dublin 2, D02 H638 through the HRB Collaborative Doctoral Awards under Grant CDA-2018-005 ("Right Care" Programme). The funder has no role in the study design, collection, management, analysis, interpretation of the data or writing of the protocol.

\section{Availability of data and materials}

Non-applicable.

\section{Declarations}

\section{Ethics approval and consent to participate}

Ethics approval was obtained from the HSE Mid-Western Area Research Ethics Committee Reference number (088/2020). Explicit informed consent will be obtained from all participants or proxy consent from the next of kin.

\section{Consent for publication}

Non-applicable.

\section{Competing interests}

The authors declare no competing interests.

\section{Author details}

${ }^{1}$ School of Allied Health, Faculty of Education and Health Sciences, Ageing Research Centre, Health Research Institute, University of Limerick, Limerick, Ireland. ${ }^{2}$ Department of Ageing and Therapeutics, University Hospital Limerick, Dooradoyle, Limerick, Ireland. ${ }^{3}$ School of Nursing and Midwifery, Faculty of Education and Health Sciences, University of Limerick, Limerick, Ireland. ${ }^{4}$ Department of Sport, Exercise \& Nutrition, School of Science \& Computing, Galway-Mayo Institute of Technology, Dublin Road, Galway, Ireland. ${ }^{5}$ Trinity Institute of Neurosciences, School of Medicine, Trinity College Dublin, Dublin, Ireland. ${ }^{6}$ Limerick EM Education Research Training (ALERT), Emergency Department, University Hospital Limerick, Dooradoyle, Limerick, Ireland.

Received: 20 September 2021 Accepted: 28 November 2021 Published online: 03 January 2022

\section{References}

1. Hwang U, Hastings SN, Ramos K. Improving emergency department discharge care with telephone follow-up. Does it connect? J Am Geriatr Soc. 2018;66(3):436-8.

2. Goldberg EM, Resnik L, Marks SJ, Merchant RC. GAPcare: the geriatric acute and post-acute fall prevention intervention-a pilot investigation of an emergency department-based fall prevention program for community-dwelling older adults. Pilot Feasibility Stud. 2019;5(1):106.

3. Ukkonen M, Jämsen E, Zeitlin R, Pauniaho S-L. Emergency department visits in older patients: a population-based survey. BMC Emerg Med. 2019;19(1):20.

4. Preston L, van Oppen JD, Conroy SP, Ablard S, Buckley Woods H, Mason SM. Improving outcomes for older people in the emergency department: 
a review of reviews. Emerg Med J. 2021;38:882-8. https://doi.org/10.1136/ emermed-2020-209514.

5. Burkett E, Martin-Khan MG, Scott J, Samanta M, Gray LC. Trends and predicted trends in presentations of older people to Australian emergency departments: effects of demand growth, population aging and climate change. Aust Health Rev. 2017;41(3):246-53.

6. Hoon LS, Mackey S, Hong-Gu H. Elderly patients' experiences of care received in the emergency department: a systematic review. JBI Evid Synth. 2012;10(23):1363-409.

7. Beaton K, Grimmer K. Tools that assess functional decline: systematic literature review update. Clin Interv Aging. 2013;8:485.

8. Ellis G, Marshall T, Ritchie C. Comprehensive geriatric assessment in the emergency department. Clin Interv Aging. 2014;9:2033.

9. Carpenter CR, Shelton E, Fowler S, Suffoletto B, Platts-Mills TF, Rothman RE, et al. Risk factors and screening instruments to predict adverse outcomes for undifferentiated older emergency department patients: a systematic review and meta-analysis. Acad Emerg Med. 2015;22(1):1-21.

10. Hastings $S N$, Heflin MT. A systematic review of interventions to improve outcomes for elders discharged from the emergency department. Acad Emerg Med. 2005;12(10):978-86.

11. Lowthian J, Straney LD, Brand CA, Barker AL, Smit PV, Newnham H, et al. Unplanned early return to the emergency department by older patients: the Safe Elderly Emergency Department Discharge (SEED) project. Age Ageing. 2016;45(2):255-61

12. Nagurney JM, Fleischman W, Han L, Leo-Summers L, Allore HG, Gill TM. Emergency department visits without hospitalization are associated with functional decline in older persons. Ann Emerg Med. 2017;69(4):426-33.

13. Provencher V, Sirois MJ, Ouellet MC, Camden S, Neveu X, Allain-Boulé N, et al. Decline in activities of daily living after a visit to a Canadian emergency department for minor injuries in independent older adults: are frail older adults with cognitive impairment at greater risk? J Am Geriatr Soc. 2015;63(5):860-8.

14. Hwang U, Dresden SM, Rosenberg MS, Garrido MM, Loo G, Sze J, et al. Geriatric emergency department innovations: transitional care nurses and hospital use. J Am Geriatr Soc. 2018;66(3):459-66.

15. Malik M, Moore Z, Patton D, O'Connor T, Nugent L. The impact of geriatric focused nurse assessment and intervention in the emergency department: a systematic review. Int Emerg Nurs. 2018;37:52-60.

16. Hughes JM, Freiermuth CE, Shepherd-Banigan M, Ragsdale L, Eucker SA, Goldstein K, et al. Emergency department interventions for older adults: a systematic review. J Am Geriatr Soc. 2019;67(7):1516-25.

17. Lowthian JA, McGinnes RA, Brand CA, Barker AL, Cameron PA. Discharging older patients from the emergency department effectively: a systematic review and meta-analysis. Age Ageing. 2015;44(5):761-70.

18. Conroy SP, Stevens T, Parker SG, Gladman JRF. A systematic review of comprehensive geriatric assessment to improve outcomes for frail older people being rapidly discharged from acute hospital: 'interface geriatrics'. Age Ageing. 2011;40(4):436-43.

19. Häseler-Ouart K, Arefian H, Hartmann M, Kwetkat A. Geriatric assessment for older adults admitted to the emergency department: a systematic review and meta-analysis. Exp Gerontol. 2021;144:111184. https://doi.org/ 10.1016/j.exger.2020.111184

20. Craig P, Dieppe P, Macintyre S, Michie S, Nazareth I, Petticrew M. Developing and evaluating complex interventions: the new Medical Research Council guidance. BMJ. 2008;337:a1655.

21. Ellis $\mathrm{G}$, Gardner M, Tsiachristas A, Langhorne P, Burke O, Harwood RH, et al. Comprehensive geriatric assessment for older adults admitted to hospital. Cochrane Database Syst Rev. 2017;9:CD006211.

22. Ekdahl AW, Sjöstrand F, Ehrenberg A, Oredsson S, Stavenow L, Wisten A, et al. Frailty and comprehensive geriatric assessment organized as CGAward or CGA-consult for older adult patients in the acute care setting: a systematic review and meta-analysis. Eur Geriatr Med. 2015;6(6):523-40.

23. Ellis $G$, Langhorne P. Comprehensive geriatric assessment for older hospital patients. Br Med Bull. 2005:71(1):45-59.

24. Harding S. Comprehensive geriatric assessment in the emergency department. Age Ageing. 2020;49(6):936-8.

25. Jay S, Whittaker P, Mcintosh J, Hadden N. Can consultant geriatrician led comprehensive geriatric assessment in the emergency department reduce hospital admission rates? A systematic review. Age Ageing. 2017:46(3):366-72.

26. Cassarino M, Robinson K, Quinn R, Naddy B, O'Regan A, Ryan D, et al. Impact of early assessment and intervention by teams involving health and social care professionals in the emergency department: a systematic review. PLoS One. 2019;14(7):e0220709.

27. Wylie K, Crilly J, Toloo G, FitzGerald G, Burke J, Williams G, et al. Review article: Emergency department models of care in the context of care quality and cost: a systematic review. Emerg Med Aust. 2015;27(2):95-101.

28. James K, Jones D, Kempenaar L, Preston J, Kerr S. Occupational therapy and emergency departments: a critical review of the literature. Br J Occup Ther. 2016;79(8):459-66.

29. Forster A, Young J. Community rehabilitation for older people: day hospital or home-based services? Age Ageing. 2011;40(1):2-4.

30. Resnick B, Galik E, Boltz M. Function focused care approaches: literature review of progress and future possibilities. J Am Med Dir Assoc. 2013;14(5):313-8

31. Izquierdo M, Merchant RA, Morley JE, Anker SD, Aprahamian I, Arai $\mathrm{H}$, et al. International exercise recommendations in older adults (ICFSR): expert consensus guidelines. J Nutr Health Aging. 2021;25:824-53. https://doi.org/10.1007/s12603-021-1665-8.

32. Wilhelmson K, Duner A, Eklund K, Gosman-Hedström G, Blomberg S, Hasson $\mathrm{H}$, et al. Design of a randomized controlled study of a multi-professional and multidimensional intervention targeting frail elderly people. BMC Geriatr. 2011;11(1):24.

33. Huang $\mathrm{H}-\mathrm{H}$, Chang JC-Y, Tseng C-C, Yang Y-J, Fan J-S, Chen Y-C, et al. Comprehensive geriatric assessment in the emergency department for the prediction of readmission among older patients: a 3-month follow-up study. Arch Gerontol Geriatr. 2021:92:104255.

34. LaMantia MA, Platts-Mills TF. Bending the curve of health trajectories for older adults discharged from the emergency department. Ann Emerg Med. 2017:69(4):434-6.

35. Samaras NMD, Chevalley TMD, Samaras DMD, Gold GMD. Older patients in the Emergency department: a review. Ann Emerg Med. 2010;56(3):261-9.

36. Kessler C, Williams MC, Moustoukas JN, Pappas C. Transitions of care for the geriatric patient in the emergency department. Clin Geriatr Med. 2013:29(1):49-69.

37. Mooijaart SP, Nickel CH, Conroy SP, Lucke JA, van Tol LS, Olthof M, et al. A European research agenda for geriatric emergency medicine: a modified Delphi study. Eur Geriatr Med. 2021;12(2):413-22.

38. Dufour I, Chouinard M-C, Dubuc N, Beaudin J, Lafontaine S, Hudon C. Factors associated with frequent use of emergency-department services in a geriatric population: a systematic review. BMC Geriatr. 2019;19(1):1-9.

39. Cadogan MP, Phillips LR, Ziminski CE. A perfect storm: care transitions for vulnerable older adults discharged home from the emergency department without a hospital admission. The Gerontologist. 2014;56(2):326-34

40. Chan AW, Tetzlaff JM, Altman DG, Laupacis A, Gøtzsche PC, Krleža-Jerić K, et al. SPIRIT 2013 statement: defining standard protocol items for clinical trials. Ann Intern Med. 2013;158(3):200-7.

41. Moore GF, Audrey S, Barker M, Bond L, Bonell C, Hardeman W, et al. Process evaluation of complex interventions: Medical Research Council guidance. BMJ. 2015;350:h1258. https://doi.org/10.1136/bmj.h1258.

42. Thabane L, Ma J, Chu R, Cheng J, Ismaila A, Rios LP, et al. A tutorial on pilot studies: the what, why and how. BMC Med Res Methodol. 2010;10(1):1.

43. Arain M, Campbell MJ, Cooper CL, Lancaster GA. What is a pilot or feasibility study? A review of current practice and editorial policy. BMC Med Res Methodol. 2010;10(1):1-7.

44. Browne RH. On the use of a pilot sample for sample size determination. Stat Med. 1995;14(17):1933-40.

45. Lancaster GA, Dodd S, Williamson PR. Design and analysis of pilot studies: recommendations for good practice. J Eval Clin Pract. 2004;10(2):307-12.

46. Griffin A, O'Neill A, O'Connor M, Ryan D, Tierney A, Galvin R. The prevalence of malnutrition and impact on patient outcomes among older adults presenting at an Irish emergency department: a secondary analysis of the OPTI-MEND trial. BMC Geriatr. 2020;20(1):455.

47. Avery KNL, Williamson PR, Gamble C, O'Connell Francischetto E, Metcalfe C, Davidson P, et al. Informing efficient randomised controlled trials: exploration of challenges in developing progression criteria for internal pilot studies. BMJ Open. 2017:7(2):e013537. 
48. Parenti N, Reggiani MLB, lannone P, Percudani D, Dowding D. A systematic review on the validity and reliability of an emergency department triage scale, the Manchester Triage System. Int I Nurs Stud. 2014;51(7):1062-9.

49. McCusker J, Bellavance F, Cardin S, Trépanier S, Verdon J, Ardman O. Detection of older people at increased risk of adverse health outcomes after an emergency visit: the ISAR screening tool. J Am Geriatr Soc. 1999:47(10):1229-37.

50. Yao JL, Fang J, Lou QQ, Anderson RM. A systematic review of the identification of seniors at risk (ISAR) tool for the prediction of adverse outcome in elderly patients seen in the emergency department. Int J Clin Exp Med. 2015;8(4):4778-86.

51. Eldridge SM, Lancaster GA, Campbell MJ, Thabane L, Hopewell S, Coleman $\mathrm{CL}$, et al. Defining feasibility and pilot studies in preparation for randomised controlled trials: development of a conceptual framework. PLoS One. 2016;11(3):e0150205.

52. Salisbury C, Man M-S, Bower P, Guthrie B, Chaplin K, Gaunt DM, et al. Management of multimorbidity using a patient-centred care model: a pragmatic cluster-randomised trial of the 3D approach. Lancet. 2018;392(10141):41-50.

53. Garvey J, Connolly D, Boland F, Smith SM. OPTIMAL, an occupational therapy led self-management support programme for people with multimorbidity in primary care: a randomized controlled trial. BMC Fam Pract. 2015;16(1):1-11.

54. Kumar GS, Klein R. Effectiveness of case management strategies in reducing emergency department visits in frequent user patient populations: a systematic review. J Emerg Med. 2013;44(3):717-29.

55. Bandura A. Health promotion by social cognitive means. Health Educ Behav. 2004;31(2):143-64.

56. Sherrington C, Fairhall N, Kwok W, Wallbank G, Tiedemann A, Michaleff ZA, et al. Evidence on physical activity and falls prevention for people aged 65+ years: systematic review to inform the WHO guidelines on physical activity and sedentary behaviour. Int I Behav Nutr Phys Act. 2020;17(1):1-9.

57. Giné-Garriga M, Roqué-Fíguls M, Coll-Planas L, Sitja-Rabert M, Salvà A. Physical exercise interventions for improving performance-based measures of physical function in community-dwelling, frail older adults: a systematic review and meta-analysis. Arch Phys Med Rehabil. 2014;95(4):753-69.e3.

58. Sherrington C, Tiedemann A. Physiotherapy in the prevention of falls in older people. J Phys. 2015;61(2):54-60.

59. UK Chief Medical Officers' Physical Activity Guidelines In: Officer CM, editor. https://assets.publishing.service.gov.uk/government/uploads/ system/uploads/attachment_data/file/832868/uk-chief-medical-officersphysical-activity-guidelines.pdf2019.

60. Volkert D. Malnutrition in older adults-urgent need for action: a plea for improving the nutritional situation of older adults. Gerontology. 2013;59(4):328-33.

61. Volkert D, Beck AM, Cederholm T, Cruz-Jentoft A, Goisser S, Hooper L, et al. ESPEN guideline on clinical nutrition and hydration in geriatrics. Clin Nutr. 2019;38(1):10-47.

62. Griffin A, O'Neill A, O'Connor M, Ryan D, Tierney A, Galvin R. The prevalence of malnutrition and impact on patient outcomes among older adults presenting at an Irish emergency department: a secondary analysis of the OPTI-MEND trial. BMC Geriatr. 2020;20(1):1-11.

63. Conneely M, Boland P, O'Neill A, Byrne D, Cronin S, Quinn D, et al. A protocol for the establishment and evaluation of an older adult stakeholder panel for health services research [version 2; peer review: 2 approved]. HRB Open Res. 2020;3:1. https://doi.org/10.12688/hrbopenres.12979.2.

64. Fried TR, Bradley EH, Williams CS, Tinetti ME. Functional disability and health care expenditures for older persons. Arch Intern Med. 2001;161(21):2602-7.

65. Mahoney Fl. Functional evaluation: the Barthel index. Maryland State Med J. 1965;14(2):61-5.

66. Sainsbury A, Seebass G, Bansal A, Young JB. Reliability of the Barthel Index when used with older people. Age Ageing. 2005;34(3):228-32.

67. Herdman M, Gudex C, Lloyd A, Janssen M, Kind P, Parkin D, et al. Development and preliminary testing of the new five-level version of EQ-5D (EQ-5D-5L). Qual Life Res. 2011;20(10):1727-36.
68. Information $\mathrm{IH}$, Authority Q. Guidelines for the economic evaluation of health technologies in Ireland 2010. Dublin: Health Information and Quality Authority; 2010.

69. Chisholm D, Knapp MR, Knudsen HC, Amaddeo F, Gaite L, van Wijngaarden B. Client socio-demographic and service receipt inventoryEuropean version: development of an instrument for international research. EPSILON Study 5. European Psychiatric Services: Inputs Linked to Outcome Domains and Needs. Br J Psychiatry Suppl. 2000;39:s28-33.

70. Glick HA. Sample size and power for cost-effectiveness analysis (part 1). PharmacoEconomics. 2011;29(3):189-98.

71. O'Cathain A, Hoddinott P, Lewin S, Thomas KJ, Young B, Adamson J, et al. Maximising the impact of qualitative research in feasibility studies for randomised controlled trials: guidance for researchers. Pilot Feasibility Stud. 2015;1(1):1-13.

72. Eldridge SM, Chan CL, Campbell MJ, Bond CM, Hopewell S, Thabane $L$, et al. CONSORT 2010 statement: extension to randomised pilot and feasibility trials. BMJ. 2016;355:5239.

73. Calvert M, Blazeby J, Altman DG, Revicki DA, Moher D, Brundage MD, et al. Reporting of patient-reported outcomes in randomized trials: the CONSORT PRO extension. Jama. 2013:309(8):814-22.

74. Braun V, Clarke V. Thematic analysis; 2012.

75. Legramante JM, Morciano L, Lucaroni F, Gilardi F, Caredda E, Pesaresi $A$, et al. Frequent use of emergency departments by the elderly population when continuing care is not well established. PLoS One. 2016;11(12):e0165939.

\section{Publisher's Note}

Springer Nature remains neutral with regard to jurisdictional claims in published maps and institutional affiliations.

Ready to submit your research? Choose BMC and benefit from:

- fast, convenient online submission

- thorough peer review by experienced researchers in your field

- rapid publication on acceptance

- support for research data, including large and complex data types

- gold Open Access which fosters wider collaboration and increased citations

- maximum visibility for your research: over $100 \mathrm{M}$ website views per year

At BMC, research is always in progress.

Learn more biomedcentral.com/submissions 